DEMOGRAPHIC RESEARCH

VOLUME 42, ARTICLE 17, PAGES 497-512 PUBLISHED 12 MARCH 2020

http://www.demographic-research.org/Volumes/Vol42/17/

DOI: $10.4054 /$ DemRes.2020.42.17

Formal Relationship 30

\title{
Revivorship and life lost to mortality
}

\section{Carl P. Schmertmann}

This article is part of the Special Collection "Formal Relationships".

Guest Editors are Joshua R. Goldstein and James W. Vaupel.

(c) 2020 Carl P. Schmertmann.

This open-access work is published under the terms of the Creative Commons Attribution 3.0 Germany (CC BY 3.0 DE), which permits use, reproduction, and distribution in any medium, provided the original author(s) and source are given credit.

See https://creativecommons.org/licenses/by/3.0/de/legalcode 


\section{Contents}

$1 \quad$ Relationships $\quad 498$

2 Proofs $\quad 499$

2.1 Multiple deaths and revivals $\quad 499$

$2.2 \quad$ Life expectancy levels and gains with $R$ revivals $\quad 500$

$2.3 \quad$ Life gained from one revival: $e^{\dagger} \quad 501$

$2.4 \quad$ Generalizing $e^{\dagger}$ : Gains from higher-order revivals $\quad 502$

2.5 Changing gains as the number of revivals increases 502

2.6 Is there a limit to life expectancy gains under repeated revivals? 503

$3 \quad$ Empirical example $\quad 504$

4 Discussion $\quad 509$

$\begin{array}{ll}\text { References } & 510\end{array}$

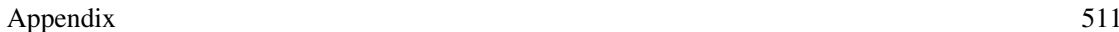




\title{
Revivorship and life lost to mortality
}

\author{
Carl P. Schmertmann ${ }^{1}$
}

\begin{abstract}
BACKGROUND

Some formal demographic models describe mortality improvement in terms of averted deaths. In such models individuals who would have died in an earlier regime are instead revived and returned to the population to face the same age-specific mortality risks as the rest of the population. A closely related literature has examined inequality in terms of the number of years of potential life that are lost to deaths.
\end{abstract}

\section{OBJECTIVE}

The paper combines several results from formal demography to illustrate the potential gains in life lived from a sequence of revivals, in which everyone is revived 0,1 , $2, \ldots$ times.

\section{CONTRIBUTION}

Mathematical analysis yields two new results: A generalization of Vaupel and CanudasRomo's $e^{\dagger}$ index to second and higher-order revivals, and an analytical expression that relates gains from revivals to the covariance of remaining life expectancy and cumulative mortality.

\footnotetext{
${ }^{1}$ Florida State University, Tallahassee, Florida, USA. Email: schmertmann @ fsu.edu.
} 


\section{Relationships}

Some demographic models (Mitra 1979; Vaupel and Yashin 1987; Finkelstein 2013) describe mortality reduction in terms of averted deaths, imagining how death rates fall when individuals are revived and restored to the population to get second, third, or higher-order chances at survival. In related papers Vaupel and colleagues (Vaupel 1986; Vaupel and Yashin 1987; Vaupel and Canudas Romo 2003) have analyzed the close connection between measures of disparity in life lived and the potential years of life that are lost to deaths. Here I combine these two threads of the literature by examining the expected gains to life from repeated revivals.

Standard models describe death as a failure-time process that removes members from a population at random ages $D$, where

$$
P(D \geq x)=\ell_{x}= \begin{cases}e^{-H_{x}} & \text { if } x \leq \omega \\ 0 & \text { if } x>\omega\end{cases}
$$

and $\omega$ represents the highest age $x$ to which anyone can survive (if there is a finite $x$ with that property), or $\omega=\infty$ (if there is not).

In this formulation $\ell_{x}$ is the probability of survival to age $x, \mu_{x}$ is the age-specific force of mortality, $H_{x}=\int_{0}^{x} \mu_{z} d z$ is cumulative mortality from birth to exact age $x$, the density of events at exact age $x$ is $\delta_{x}=\ell_{x} \mu_{x}$, and everyone experiences the event in question (death) exactly once. ${ }^{2}$

Generalizing, it is possible to think of the standard length of life as measuring the time to a first event in a nonhomogeneous Poisson process with rates $\mu_{x}$. In such a process $H_{x}$ is the expected number of events experienced by age $x$, and the probability that an individual experiences exactly $R$ events between birth and age $x$ is $\frac{e^{-H_{x}} H_{x}^{R}}{R !}=\ell_{x} \cdot\left(\frac{H_{x}^{R}}{R !}\right)$ for $R=0,1,2, \ldots$. In this alternative interpretation, individuals could remain in the population to experience second, third, and higher-order deaths.

\section{Four relationships}

In a demographic regime where each individual is granted exactly $R \in\{0,1,2, \ldots\}$ revivals, life ends at the $(R+1)$ th death, and the following relationships hold:

1. The probability of survival to exact age $x$ is $\ell_{x}^{(R)}=\ell_{x}\left(\sum_{k=0}^{R} \frac{H_{x}^{k}}{k !}\right)$.

\footnotetext{
${ }^{2}$ Time and age are continuous, but I use subscripts - e.g., $\mu_{x}$ rather than $\mu(x)$ - for brevity. I use the traditional notation $H_{x}$ for cumulative mortality; this symbol is distinct from the entropy measure $H$ that often appears in the literature on revivorship.
} 
2. The density of (final) deaths at exact age $x$ is $\delta_{x}^{(R)}=\frac{H_{x}^{R}}{R !} \delta_{x}$.

3. The gain in life expectancy from an additional $(R+1)$ th revival would be $G^{(R)}=$ $\frac{\operatorname{Cov}\left(x, H_{x}\right)}{R+1}$, where covariance is calculated over the density $\delta_{x}^{(R)}$.

4. Gains in life expectancy from consecutive revivals are related by $G^{(R+1)}=G^{(R)}+$ $\frac{\operatorname{Cov}\left(e_{x}, H_{x}\right)}{R+1}$, where covariance is calculated over the density $\delta_{x}^{(R)}$.

Relationships 1 and 2 are easily derived from basic principles of nonhomogeneous Poisson processes. Relationships 3 and 4 are, to my knowledge, new. Relationship 3 generalizes the $e^{\dagger}$ concept (Vaupel and Canudas Romo 2003) to higher-order revivals: $e^{\dagger}=G^{(0)}$ is a special case.

\section{Proofs}

\subsection{Multiple deaths and revivals}

In a nonhomogeneous Poisson process with mortality rates $\mu_{x}$ and $R$ guaranteed revivals, an individual survives to age $x$ if they have experienced $R$ or fewer events prior to age $x$ :

$$
\begin{aligned}
\ell_{x}^{(R)} & =\operatorname{Pr}[(0,1, \ldots, \mathrm{R}) \text { events prior to age } x] \\
& =\frac{e^{-H_{x}} H_{x}^{0}}{0 !}+\cdots+\frac{e^{-H_{x}} H_{x}^{R}}{R !} \\
& =\ell_{x} \cdot\left(\sum_{k=0}^{R} \frac{H_{x}^{k}}{k !}\right)
\end{aligned}
$$

Equation (1) is Relationship 1.

With $R$ guaranteed revivals, the $(R+1)$ st event removes an individual from the population. The density of (final) deaths at age $x$ is thus

$$
\delta_{x}^{(R)}=\underbrace{\frac{e^{-H_{x}} H_{x}^{R}}{R !}}_{\text {prob of exactly R events by age } x} \times \underbrace{\mu_{x}}_{\text {event intensity at age } x}=\frac{H_{x}^{R}}{R !} \delta_{x},
$$

where $\delta_{x}=\delta_{x}^{(0)}=\ell_{x} \mu_{x}$ is the customary density of life table deaths. Equation (2) is Relationship 2; it also follows from fundamental principles of Poisson processes and from the relationships between life table functions $H_{x}, \ell_{x}, \mu_{x}$, and $\delta_{x}$. 
In Equation (2), $R$ is a fixed number of revivals offered to each individual, ${ }^{3}$ and $\delta_{x}^{(R)}$ is the resulting density of (final) deaths. Final death densities with $R=0,1,2,3$ revivals would be

$$
\begin{array}{rlrl}
\delta_{x}^{(0)} & =\delta_{x} & \text { first deaths } \\
\delta_{x}^{(1)}=H_{x} \delta_{x} & \text { second deaths } \\
\delta_{x}^{(2)}=\frac{H_{x}^{2}}{2 !} \delta_{x} & \text { third deaths } \\
\delta_{x}^{(3)}=\frac{H_{x}^{3}}{3 !} \delta_{x} & \text { fourth deaths }
\end{array}
$$

respectively. All of these densities integrate to one over $[0, \omega]$. Because

$$
\delta_{x}^{(R+1)}=\frac{H_{x}}{R+1} \delta_{x}^{(R)},
$$

adding an $(R+1)$ th revival will lower the density of deaths at ages $x$ for which $H_{x}<$ $R+1$ and raise the density at ages for which $H_{x}>R+1$.

\subsection{Life expectancy levels and gains with $R$ revivals}

Define $E^{(R)}[\cdot]$ as the expectation of any age-related quantity over $\delta_{x}^{(R)}$, the density of deaths in a regime with $R$ revivals. Similarly define $\operatorname{Cov}^{(R)}[\cdot, \cdot]$ as the covariance of any two age-related quantities using density $\delta_{x}^{(R)}$.

Now use these operators to analyze life expectancy changes under repeated revivals. Life expectancy at birth is the expected age at death, so with $R$ revivals, $e_{0}$ is

$$
e_{0}^{(R)}=\int_{0}^{\omega} x \delta_{x}^{(R)} d x=E^{(R)}[x]
$$

In a regime with $R$ guaranteed revivals, the gain in life expectancy from an additional $R+1$-th revival for each individual would be

$$
G^{(R)}=e_{0}^{(R+1)}-e_{0}^{(R)}=E^{(R+1)}[x]-E^{(R)}[x] .
$$

\footnotetext{
${ }^{3}$ Some demographic models (Vaupel and Yashin 1987) treat the number of revivals as random, some (Mitra 1979) treat it as fixed, and some (Finkelstein 2013) deal with both cases.
} 
As noted by Vaupel and Canudas Romo (2003), gains in life expectancy from a revival may also be expressed in terms of the average remaining life for survivors at the time of deaths, so that

$$
G^{(R)}=\int_{0}^{\omega} e_{x} \delta_{x}^{(R)} d x=E^{(R)}\left[e_{x}\right]
$$

where $e_{x}$ represents the conventional (i.e., $R=0$ ) expectation of remaining life at exact age $x$.

\subsection{Life gained from one revival: $e^{\dagger}$}

The average amount of life lost to first deaths, which could be recovered if each individual were revived exactly one time (Vaupel and Yashin 1987; Vaupel and Canudas Romo 2003), is

$$
\begin{aligned}
e^{\dagger} & =\int_{0}^{\omega} e_{x} \delta_{x} d x \\
& =E^{(0)}\left[e_{x}\right] \\
& =E^{(1)}[x]-E^{(0)}[x] \\
& =G^{(0)}
\end{aligned}
$$

Life expectancy at birth is a weighted average of ages, and $e^{\dagger}=G^{(0)}$ measures the change in average age when the density changes from $\delta_{x}^{(0)} \rightarrow \delta_{x}^{(1)}$ - i.e., from $\delta_{x} \rightarrow H_{x} \delta_{x}$. Thus we can analyze $G^{(0)}$ using Vaupel and Zhang's (2012) result about how changes in weights affect means. Applying their main result to Equation (7) implies that

$$
e^{\dagger}=G^{(0)}=\frac{\operatorname{Cov}^{(0)}\left[x, H_{x}\right]}{E^{(0)}\left[H_{x}\right]} .
$$

We can further simplify by using the relationship $E^{(0)}\left[H_{x}\right]=1$, as derived in Appendix Equation (16). Thus

$$
e^{\dagger}=G^{(0)}=\operatorname{Cov}^{(0)}\left[x, H_{x}\right]
$$


$H_{x}$ is non-decreasing in $x$, so this covariance cannot be negative - i.e., offering first revivals could not decrease life expectancy. Potential gains in life expectancy are greater when there is higher inequality in ages at death, because covariance increases when the variance of ages at death, $E^{(0)}\left[\left(x-e_{0}^{(0)}\right)^{2}\right]$, is high.

\subsection{Generalizing $e^{\dagger}$ : Gains from higher-order revivals}

It is straightforward to generalize $e^{\dagger}$ to second, third, and higher-order revivals by combining Equations. (3) and (5) and then applying Vaupel and Zhang's (2012) result about changing averages. A change from a regime with $R$ revivals to one with $R+1$ revivals will change life expectancy at birth by $G^{(R)}=E^{(R+1)}[x]-E^{(R)}[x]$ years, with the weights changing from $\delta_{x}^{(R)} \rightarrow \frac{H_{x}}{R+1} \delta_{x}^{(R)}$. Thus

$$
\begin{aligned}
G^{(R)} & =\frac{\operatorname{Cov}^{(R)}\left[x, \frac{H_{x}}{R+1}\right]}{E^{(R)}\left[\frac{H_{x}}{R+1}\right]} \\
& =\frac{\operatorname{Cov}^{(R)}\left[x, H_{x}\right]}{E^{(R)}\left[H_{x}\right]} \\
& =\frac{\operatorname{Cov}^{(R)}\left[x, H_{x}\right]}{R+1},
\end{aligned}
$$

where the last step in Equation (10) uses appendix result (19).

Equation (10) is Relationship 3. When $R=0$, it yields the special case $e^{\dagger}=G^{(0)}=$ $\operatorname{Cov}^{(0)}\left[x, H_{x}\right]$. In all cases, the gain from an additional $(R+1)$ th revival is higher when there is more inequality/higher variance in the ages of $(R+1)$ th deaths.

\subsection{Changing gains as the number of revivals increases}

It is interesting to investigate whether there are decreasing returns to successive revivals i.e., whether the life gained from yet another revival gets smaller as the number of revivals increases. In mathematical terms, this is a question about the sign of $G^{(R+1)}-G^{(R)}$ as $R$ gets larger.

Because $G^{(R+1)}-G^{(R)}=E^{(R+1)}\left[e_{x}\right]-E^{(R)}\left[e_{x}\right]$, we can again apply Vaupel 
and Zhang's (2012) result about weighted averages. This yields

$$
\begin{aligned}
G^{(R+1)}-G^{(R)} & =\frac{\operatorname{Cov}^{(R)}\left[e_{x}, \frac{H_{x}}{R+1}\right]}{E^{(R)}\left[\frac{H_{x}}{R+1}\right]} \\
& =\frac{\operatorname{Cov}^{(R)}\left[e_{x}, H_{x}\right]}{E^{(R)}\left[H_{x}\right]} \\
& =\frac{\operatorname{Cov}^{(R)}\left[e_{x}, H_{x}\right]}{R+1} .
\end{aligned}
$$

This is Relationship 4.

From Equation (11) it is immediately possible to understand the pattern of gains from successive revivals in two specific cases. First, if $e_{x}$ declines monotonically with age - as it would with monotonically increasing mortality rates - then $\operatorname{Cov}\left(e_{x}, H_{x}\right)$ is negative for any weighting function, and gains from successive revivals are decreasing: $G^{(R+1)}<G^{(R)}$. Second, if $e_{x}$ is constant over $x-$ as it would be with identical mortality at all ages $\mu_{x}=\mu^{*}$ - then the covariance term in Equation (11) is zero for any weighting function, and life gained is identical for each successive revival: $G^{(R+1)}=G^{(R)}$.

With high numbers of revivals $R$, the death distribution $\delta_{x}^{(R)}$ will be concentrated at very high ages, and the $e_{x}$ and $H_{x}$ values that matter most in Equation (11) will be those for very high ages $x$. In practice this means that either of the specific situations discussed in the last paragraph may be approximately true. If mortality rates approach some asymptotic value $\mu_{x} \rightarrow \mu^{*}$ at very high ages, then $e_{x} \rightarrow \frac{1}{\mu^{*}}$ and gains from further high-order revivals $G^{(R)}, G^{(R+1)}, \ldots$ will tend to become identical. In contrast, if mortality continues to increase with $x$, then $e_{x} \rightarrow 0$, covariances in Equation (11) become negative, and gains will be smaller for each successive revival. It is also theoretically possible for $G^{(R)}$ to increase with $R$ if there are sharp increases in $e_{x}$ after reaching high ages, but for human populations that is extremely unlikely.

\subsection{Is there a limit to life expectancy gains under repeated revivals?}

Because

$$
G^{(R)}=E^{(R)}\left[e_{x}\right]=\int_{0}^{\omega} e_{x} \delta_{x}^{(R)} d x=\int_{0}^{\omega} e_{x} \frac{H_{x}^{R}}{R !} \delta_{x} d x
$$


the cumulative gain from $R$ guaranteed revivals would be

$$
\sum_{r=0}^{R-1} G^{(r)}=\int_{0}^{\omega} e_{x}\left[1+H_{x}+\frac{H_{x}^{2}}{2 !}+\cdots+\frac{H_{x}^{R-1}}{(R-1) !}\right] \delta_{x} d x .
$$

As the number of revivals grows, the term in square brackets approaches a limit of $e^{H_{x}}=$ $\frac{1}{\ell_{x}}$, so the cumulative gain approaches

$$
\begin{aligned}
\lim _{R \rightarrow \infty}\left(\sum_{r=0}^{R-1} G^{(r)}\right) & =\int_{0}^{\omega} e_{x}\left[e^{H_{x}}\right] \delta_{x} d x \\
& =\int_{0}^{\omega} e_{x} \mu_{x} d x=\int_{0}^{\omega}\left(1+e_{x}^{\prime}\right) d x=\omega-e_{0} .
\end{aligned}
$$

Thus, if there is a finite age $\omega$ beyond which survival is impossible, then there is an upper limit on the total gains in life expectancy available through repeated revivals, and the series $\left(G^{(0)}+G^{(1)}+G^{(2)}+\cdots\right)$ converges to $\omega-e_{0}$. If there is no such age, then $\left(G^{(0)}+G^{(1)}+G^{(2)}+\cdots\right)$ does not converge and there is no upper limit to the cumulative gains from repeated revivals. Note that the non-convergence result for $\omega=\infty$ applies to any pattern of mortality for which there are non-zero probabilities of survival to all ages, no matter how small those probabilities are. ${ }^{4}$

\section{Empirical example}

Consider an example based on mortality rates of Danish females in 1970 from the Human Mortality Database (HMD 2014). Figure 1 illustrates a (log) mortality schedule based on the HMD rates, graduated to intervals of 0.1 years and extrapolated to ages above 100 . At the highest ages, I assume that either (1) log mortality rates approach an asymptotic limit of zero at high ages, as in a Kannisto model (Thatcher, Kannisto, and Vaupel 1998), or (2) $\log$ mortality rates increase linearly at ages 100 -plus, as in a Gompertz model. ${ }^{5}$ Without revivals, $e_{0}^{(0)}=76.3$ in both models.

\footnotetext{
${ }^{4}$ A more intuitive way to understand the converence/non-convergence results is to note that from Equation (1), $\lim _{R \rightarrow \infty}\left[\ell_{x}^{(R)}\right]=1$ for any $x<\omega$. Thus $\int_{0}^{\omega} \ell_{x}^{(R)} d x \rightarrow \omega$, which is finite if and only if $\omega<\infty$.

${ }^{5}$ Mortality rates at ages $0-100$ in this example are derived from Denmark 1970 data, but because of smoothing and discretization, they are not identical.
} 
Figure 1: Two mortality schedules derived from Denmark 1970 female data, smoothed to intervals of 0.1 years. At ages 100-plus mortality is extrapolated under two alternative assumptions: A continued linear increase in log rates (Gompertz) or an asymptotic limit (Kannisto)

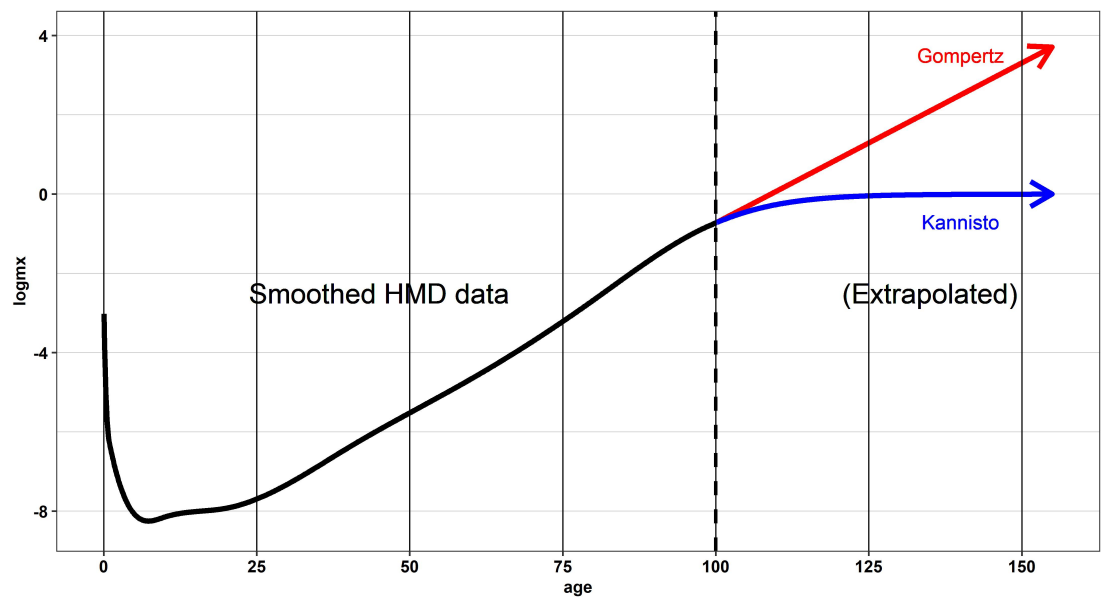

Figure 2 displays the distributions of ages at death $\delta_{x}^{(R)}$ with different numbers of revivals $-R \in\{0,1,5,10,20,30\}$ - under the Kannisto and Gompertz patterns of oldest-age mortality. With the Kannisto pattern (nearly constant mortality risks after age 100), revivals lead to ever-higher life expectancies, but also to increasing inequality in the lengths of individual lives for higher-order revivals. With the Gompertz pattern, successive revivals must climb an ever-steeper hill as lives are lengthened; as a result, gains $G^{(R)}$ become smaller, but uncertainty about length of life decreases.

Figure 3 reports a more complete set of results, for all $R$ values between 0 and 30. Table 1 contains the same results in numerical form. The left panel of Figure 3 displays levels of life expectancy $e_{0}^{(R)}$ as a function of the number of revivals $R$ for $R$ values $0,1, \ldots, 30$. The right panel displays the gains from one additional revival $G^{(R)}$. As suggested by the densities in Figure 2, life expectancies rise more rapidly and more linearly in the Kannisto case, with constant oldest-age mortality.

The patterns of gains from further revivals in the right panel show that, as expected from Relationship 4, gains from successive revivals stabilize when oldest-age mortality rates tend toward a positive asymptotic limit as age increases (Kannisto case). This occurs because as one moves the death densities to high ages in the Kannisto case, $e_{x}$ behaves 
Figure 2: $\quad$ Death densities $\delta_{x}^{(R)}$ with different numbers of revivals at the mortality rates in Figure 1 (excludes points where density $<10^{-5}$ )

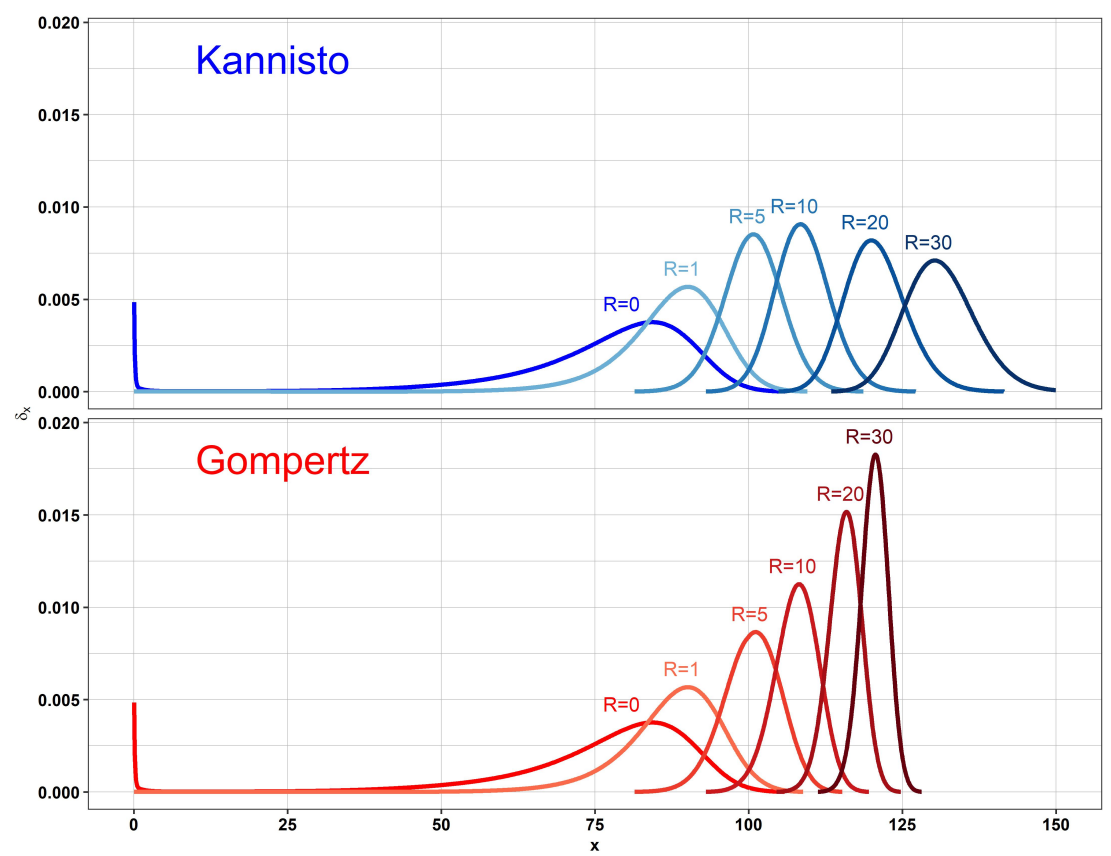

more and more like a constant $e_{x} \approx \frac{1}{\mu^{*}}=1$. Consequently $\operatorname{Cov}^{(R)}\left[e_{x}, H_{x}\right] \rightarrow 0$ and gains stabilize as $G^{(R)} \rightarrow 1$.

In contrast, when oldest-age mortality rates increase monotonically with age (Gompertz case), gains from successive revivals become smaller with $R$ because $\operatorname{Cov}^{(R)}\left[e_{x}, H_{x}\right]<0$, so that $G^{(R+1)}<G^{(R)}$. Interestingly, although gains from successive revivals decline toward zero in the Gompertz case, they do so slowly enough that the cumulative gain $G^{(0)}+G^{(1)}+G^{(2)}+\cdots$ does not converge to any limit. As shown by Equation (14), the lower curve in the left panel of Figure 3 will continue increasing with $R$ indefinitely, although at an ever-slower rate. 
Figure 3: $\quad$ Life expectancy with different numbers of revivals under alternative models of oldest-age mortality. Left panel illustrates $e_{0}^{(R)}$ levels for $R=0,1, \ldots, 30$ revivals. Right panel illustrates gains from additional revivals $G^{(R)}$.
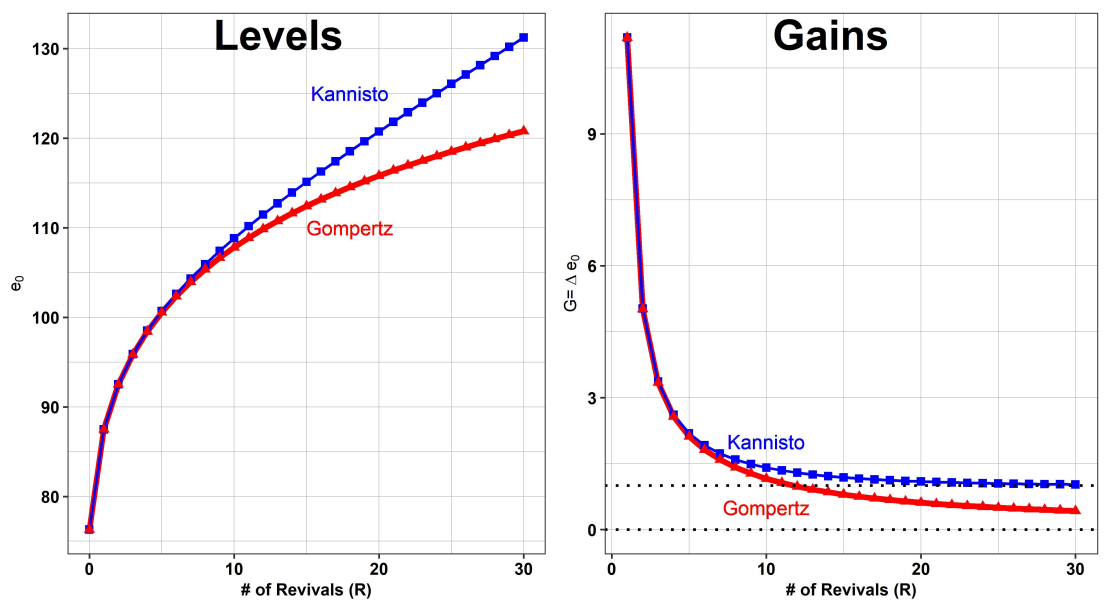
Schmertmann: Revivorship and life lost to mortality

Table 1: Life expectancy at birth and gains from different numbers of revivals $R$. Numerical approximations may include small rounding errors. Last line shows limiting values as $\boldsymbol{R} \rightarrow \infty$.

\begin{tabular}{|c|c|c|c|c|c|c|}
\hline \multirow[b]{2}{*}{$\mathbf{R}$} & \multicolumn{2}{|c|}{$e_{0}^{(R)}$} & \multicolumn{2}{|c|}{$\frac{\operatorname{Cov}^{(R)}\left[x, H_{x}\right]}{R+1}=$} & \multicolumn{2}{|c|}{$\frac{\operatorname{Cov}^{(R)}\left[e_{x}, H_{x}\right]}{R+1}=$} \\
\hline & Kannisto & Gompertz & Kannisto & Gompertz & Kannisto & Gompertz \\
\hline 0 & 76.30 & 76.30 & 11.19 & 11.19 & -6.17 & -6.18 \\
\hline 1 & 87.49 & 87.49 & 5.02 & 5.02 & -1.67 & -1.68 \\
\hline 2 & 92.52 & 92.51 & 3.36 & 3.34 & -0.76 & -0.78 \\
\hline 3 & 95.88 & 95.85 & 2.61 & 2.57 & -0.43 & -0.45 \\
\hline 4 & 98.50 & 98.43 & 2.19 & 2.12 & -0.27 & -0.31 \\
\hline 5 & 100.68 & 100.55 & 1.91 & 1.81 & -0.19 & -0.23 \\
\hline 6 & 102.60 & 102.36 & 1.73 & 1.58 & -0.14 & -0.18 \\
\hline 7 & 104.33 & 103.95 & 1.59 & 1.41 & -0.10 & -0.14 \\
\hline 8 & 105.92 & 105.37 & 1.49 & 1.27 & -0.08 & -0.12 \\
\hline 9 & 107.41 & 106.64 & 1.41 & 1.15 & -0.06 & -0.10 \\
\hline 10 & 108.82 & 107.80 & 1.34 & 1.06 & -0.05 & -0.08 \\
\hline 11 & 110.17 & 108.86 & 1.29 & 0.97 & -0.04 & -0.07 \\
\hline 12 & 111.46 & 109.84 & 1.25 & 0.90 & -0.04 & -0.06 \\
\hline 13 & 112.71 & 110.76 & 1.21 & 0.84 & -0.03 & -0.05 \\
\hline 14 & 113.93 & 111.61 & 1.18 & 0.79 & -0.02 & -0.05 \\
\hline 15 & 115.11 & 112.41 & 1.16 & 0.74 & -0.02 & -0.04 \\
\hline 16 & 116.27 & 113.16 & 1.14 & 0.70 & -0.02 & -0.04 \\
\hline 17 & 117.41 & 113.87 & 1.12 & 0.66 & -0.02 & -0.03 \\
\hline 18 & 118.53 & 114.55 & 1.10 & 0.63 & -0.01 & -0.03 \\
\hline 19 & 119.64 & 115.19 & 1.09 & 0.60 & -0.01 & -0.03 \\
\hline 20 & 120.73 & 115.80 & 1.08 & 0.57 & -0.01 & -0.03 \\
\hline 21 & 121.81 & 116.39 & 1.07 & 0.55 & -0.01 & -0.02 \\
\hline 22 & 122.89 & 116.95 & 1.06 & 0.52 & -0.01 & -0.02 \\
\hline 23 & 123.95 & 117.49 & 1.05 & 0.50 & -0.01 & -0.02 \\
\hline 24 & 125.00 & 118.01 & 1.05 & 0.48 & -0.01 & -0.02 \\
\hline 25 & 126.05 & 118.51 & 1.04 & 0.46 & -0.01 & -0.02 \\
\hline 26 & 127.10 & 118.99 & 1.04 & 0.45 & 0.00 & -0.02 \\
\hline 27 & 128.13 & 119.46 & 1.03 & 0.43 & 0.00 & -0.01 \\
\hline 28 & 129.17 & 119.91 & 1.03 & 0.42 & 0.00 & -0.01 \\
\hline 29 & 130.20 & 120.35 & 1.03 & 0.40 & 0.00 & -0.01 \\
\hline 30 & 131.22 & 120.77 & 1.02 & 0.39 & 0.00 & -0.01 \\
\hline$\infty$ & $\infty$ & $\infty$ & 1 & 0 & 0 & 0 \\
\hline
\end{tabular}




\section{Discussion}

Analysis of potential life gained from repeatedly averting deaths is highly speculative but demographically and mathematically interesting. Considering progress against mortality as the outcome of repeated minimal repairs (Finkelstein 2005), in which individuals who would otherwise have died are instead revived and directly returned to the living population in their pre-death state, produces interesting insights into the potential consequences of sustained mortality decline.

Some of these results have been analyzed and discussed in earlier demographic contributions (Vaupel and Yashin 1987; Vaupel, Zhang, and van Raalte 2011; Finkelstein 2005, 2013). Most important for the exposition here, Vaupel and others have shown how the potential gains from revival $\left(e^{\dagger}\right)$ are related to disparity in the distribution of length of life: The more unequal the distribution of current ages at death, the larger the potential gains from averting (first) deaths.

Here I present an exposition that includes these earlier results about revivals, disparities, and gains in life expectancy and also allows generalization to a sequence of repeated revivals. This analysis produces (1) interesting relationships between gains from revivals and covariances of standard life table functions, (2) a finding that repeated revivals could lead to increases in the dispersion of length of life if mortality rates approach an asymptotic limit at very high ages, and (3) insights about the (lack of) limits on life expectancy with an unlimited number of revivals.

The model explored here highlights the same fundamental questions that any analysis of mortality improvement must must confront: Can life expectancy continue to increase, are there decreasing gains to those improvements, and what are the implications for inequality in life lived? 


\section{References}

Finkelstein, M. (2013). Lifesaving, delayed deaths and cure in mortality modeling. Theoretical Population Biology 83: 15-19. doi:10.1016/j.tpb.2012.10.005.

Finkelstein, M. (2005). Lifesaving explains mortality decline with time. Mathematical Biosciences 196(2): 187-197. doi:10.1016/j.mbs.2005.04.004.

HMD (2014). Human Mortality Database [electronic resource]. Berkeley: University of California, Rostock: Max Planck Institute for Demographic Research. mortality.org.

Mitra, S. (1979). The effects of extra chances to live on life table functions. Theoretical Population Biology 16(3): 315-322. doi:10.1016/0040-5809(79)90020-0.

Thatcher, R.A., Kannisto, V., and Vaupel, J.W. (1998). The force of mortality at ages 80 to 120. Odense: Odense University Press.

Vaupel, J.W. and Canudas Romo, V. (2003). Decomposing change in life expectancy: A bouquet of formulas in honor of Nathan Keyfitz's $90^{\text {th }}$ birthday. Demography 40(2): 201-216. doi:10.1353/dem.2003.0018.

Vaupel, J.W. and Yashin, A.I. (1987). Repeated resuscitation: How lifesaving alters life tables. Demography 24(1): 123-135. doi:10.2307/2061512.

Vaupel, J.W. and Zhang, Z. (2012). The difference between alternative averages. Demographic Research S8(15): 419-428. doi:10.4054/DemRes.2012.27.15.

Vaupel, J.W., Zhang, Z., and van Raalte, A.A. (2011). Life expectancy and disparity: An international comparison of life table data. BMJ Open 1(1). doi:10.1136/bmjopen2011-000128.

Vaupel, J. (1986). How change in age-specific mortality affects life expectancy. Population Studies 40(1): 147-157. doi:10.1080/0032472031000141896. 


\section{Appendix}

Derivation: $E^{(R)}\left[H_{x}\right]=R+1$

Note that

$$
E^{(0)}\left[H_{x}\right]=\int_{0}^{\omega} H_{x} \delta_{x} d x
$$

can be integrated by parts using $u=H_{x}, d u=\mu_{x} d x, d v=\delta_{x} d x, v=-\ell_{x}$ as

$$
\begin{aligned}
E^{(0)}\left[H_{x}\right] & \left.=-\ell_{x} H_{x}\right]_{x=0}^{\omega}+\int_{0}^{\omega} \mu_{x} \ell_{x} d x \\
& =0+\int_{0}^{\omega} \delta_{x} d x \\
& =0+1=1 .
\end{aligned}
$$

The first term has a limit of zero because $\ell_{x} H_{x}=e^{-H_{x}} H_{x}=\frac{H_{x}}{e^{H_{x}}}$, which has a limit of zero by L'Hôpital's rule. Thus $E^{(0)}\left[H_{x}\right]=1$.

Integrating

$$
E^{(R)}\left[H_{x}\right]=\int_{0}^{\omega} H_{x} \frac{H_{x}^{R}}{R !} \delta_{x} d x
$$

by parts in the same manner, with $u=\frac{H_{x}^{R+1}}{R !}, d u=\frac{R+1}{R !} H_{x}^{R} \mu_{x} d x, d v=\delta_{x} d x, v=-\ell_{x}$, yields

$$
\begin{aligned}
E^{(R)}\left[H_{x}\right] & \left.=-\ell_{x} \frac{H_{x}^{R+1}}{R !}\right]_{x=0}^{\omega}+\frac{R+1}{R} \int_{0}^{\omega} H_{x} \frac{H_{x}^{R-1}}{(R-1) !} \delta_{x} d x \\
& =0+\left(\frac{R+1}{R}\right) \cdot E^{(R-1)}\left[H_{x}\right] .
\end{aligned}
$$


Schmertmann: Revivorship and life lost to mortality

Repeated application of the recursive relationship in Equation (18) shows that

$$
\begin{aligned}
E^{(R)}\left[H_{x}\right] & =\left(\frac{R+1}{R}\right) E^{(R-1)}\left[H_{x}\right] \\
& =\left(\frac{R+1}{R-1}\right) E^{(R-2)}\left[H_{x}\right] \\
& =\cdots \\
& =\left(\frac{R+1}{1}\right) E^{(0)}\left[H_{x}\right] \\
& =R+1 .
\end{aligned}
$$

